\title{
Phenotypic alterations in pancreatic lymph node stromal cells from human donors with type 1 diabetes and NOD mice
}

\author{
Jorge Postigo-Fernandez ${ }^{1,2,3} \cdot$ Donna L. Farber ${ }^{1,4,5} \cdot$ Rémi J. Creusot ${ }^{1,2,3}$
}

Received: 10 May 2019 / Accepted: 15 July 2019/Published online: 5 September 2019

(C) Springer-Verlag GmbH Germany, part of Springer Nature 2019

\begin{abstract}
Aims/hypothesis Tolerance induction in lymph nodes can be mediated by both haematopoietic cells (e.g. specific dendritic cells subsets) and by non-haematopoietic cells (e.g. lymph node stromal cells [LNSCs]) when they present peripheral tissue antigens to autoreactive T cells. LNSCs normally regulate T cell trafficking and survival and help to maintain peripheral tolerance by exerting immunosuppressive effects. However, whether autoimmunity can be associated with defective tolerogenic functions of LNSCs is unknown and studies aimed at characterising LNSCs in humans are lacking. We hypothesised that dysregulated T cell responses in pancreatic lymph nodes (PLNs) from donors with type 1 diabetes and from NOD mice may be associated with altered LNSC function.

Methods We analysed PLNs from donors with type 1 diabetes and NOD mice for LNSC distribution and phenotype using flow cytometry. We assessed the expression of tolerance-related genes in different subsets of LNSCs from human donors, as well as in a population of dendritic cells enriched in autoimmune regulator (AIRE) ${ }^{+}$cells and identified as HLA-DR ${ }^{\text {high }} \mathrm{CD} 45^{\text {low }}$.

Results The relative frequency of different LNSC subsets was altered in both donors with type 1 diabetes and NOD mice, and both MHC class II and programmed death-ligand 1 (PD-L1) expression were upregulated in human type 1 diabetes. Tolerancerelated genes showed similar expression profiles between mouse and human LNSCs at steady state but were generally upregulated in the context of human type 1 diabetes, while, at the same time, many such genes were downregulated in the AIREenriched dendritic cell population.

Conclusion/interpretation Our study shows that LNSCs are substantially altered in type 1 diabetes, but, surprisingly, they exhibit an enhanced tolerogenic phenotype along with increased antigen-presenting potential, which may indicate an attempt to offset dendritic cell-related tolerogenic defects in tolerance. Thus, LNSCs could constitute alternative therapeutic targets in which to deliver antigens to help re-establish tolerance and prevent or treat type 1 diabetes.

Data availability All data generated or analysed during this study are included in the published article (and its online supplementary files). Biomark gene expression data were deposited on the Mendeley repository at https://data.mendeley.com/datasets/ d9rdzdmvyf/1. Any other raw datasets are available from the corresponding author on reasonable request. No applicable resources were generated or analysed during the current study.
\end{abstract}

Keywords Beta cell antigens $\cdot$ Dendritic cells $\cdot$ Lymph node stromal cells $\cdot$ Pancreatic lymph nodes $\cdot$ Tolerance $\cdot$ Type 1 diabetes

Electronic supplementary material The online version of this article (https://doi.org/10.1007/s00125-019-04984-w) contains peer-reviewed but unedited supplementary material, which is available to authorised users.

Rémi J. Creusot rjc2150@ columbia.edu

1 Columbia Center for Translational Immunology, Columbia University Medical Center, 650 W. 168th Street, New York, NY 10032, USA

2 Department of Medicine, Columbia University Medical Center, New York, NY, USA
3 Naomi Berrie Diabetes Center, Columbia University Medical Center, New York, NY, USA

4 Department of Surgery, Columbia University Medical Center, New York, NY, USA

5 Department of Microbiology \& Immunology, Columbia University Medical Center, New York, NY, USA 


\section{Research in context}

\section{What is already known about this subject?}

- Lymph node stromal cells (LNSCs) can endogenously express and present self-antigens to T lymphocytes and induce tolerogenic responses through the expression of inhibitory molecules

- Conventional antigen-presenting cells, including dendritic cells, show defects in maintaining tolerance in type 1 diabetes

- There is an imbalance of T cell responses in the pancreatic lymph nodes (PLNs) of individuals with type 1 diabetes

\section{What is the key question?}

- $\quad$ Are LNSCs phenotypically and functionally altered in PLNs in type 1 diabetes?

\section{What are the new findings?}

- $\quad$ LNSC subsets are altered in their distribution in PLNs in the context of type 1 diabetes

- Most LNSC subsets exhibit increased expression of MHC-II and programmed death-ligand 1 (PD-L1) in PLNs from donors with type 1 diabetes

- $\quad$ LNSCs have an overall increase, rather than a reduction, in the expression of tolerance-related genes

How might this impact on clinical practice in the foreseeable future?

- Targeting LNSCs as part of antigen-specific therapies may constitute a novel and possibly more efficient way to reestablish tolerance for the treatment or prevention of type 1 diabetes

$\begin{array}{ll}\text { Abbreviations } \\ \text { AAb } & \text { Autoantibody } \\ \text { AIRE } & \text { Autoimmune regulator } \\ \text { APC } & \text { Antigen-presenting cell } \\ \text { BEC } & \text { Blood (vascular) endothelial cell } \\ \text { DNC } & \text { Double-negative cell } \\ \text { FEZF2 } & \text { FEZ family zinc finger } 2 \\ \text { FRC } & \text { Fibroblastic reticular cell } \\ \text { LEC } & \text { Lymphatic endothelial cell } \\ \text { LNSC } & \text { Lymph node stromal cell } \\ \text { MFI } & \text { Mean fluorescence intensity } \\ \text { NOR } & \text { Non-obese diabetes-resistant } \\ \text { nPOD } & \text { Network of Pancreatic Organ Donors with Diabetes } \\ \text { PD-L1 } & \text { Programmed death-ligand 1 } \\ \text { PDPN } & \text { Podoplanin } \\ \text { PLN } & \text { Pancreatic lymph nodes } \\ \text { TRA } & \text { Tissue-restricted antigen }\end{array}$

\section{Introduction}

Type 1 diabetes results from a breakdown in central and/or peripheral tolerance to specific beta cell antigens [1]. Both genetic and environmental factors contribute to defective induction or maintenance of immune tolerance by affecting the function of lymphocytes [2, 3] and antigen-presenting cells (APCs) [4]. Pancreatic lymph nodes (PLNs) drain islets and therefore play a central role in the pathogenesis of type 1 diabetes. In NOD mice, diabetogenic T cells are stimulated by islet-derived antigens in PLNs but not in other lymph nodes [5]. In individuals with type 1 diabetes, evidence for diabetogenic $\mathrm{T}$ cell stimulation and defective regulatory $\mathrm{T}$ cell function has been found in PLNs [3, 6].

Dendritic cells constitute the most studied population of APCs that have the ability to acquire self-antigens within tissues or lymph nodes draining those tissues. Therefore, essentially all studies that aimed at determining whether defective tolerance is associated with alterations in APC function have been focused on dendritic cells and, to some extent, macrophages, all of which are professional APCs with known involvement in type 1 diabetes [4]. However, a number of nonprofessional (non-haematopoietic) APCs with the ability to mediate immune tolerance have been described [7, 8], and whether these play a role in type 1 diabetes is unknown. Among them, lymph node stromal cells (LNSCs) are crucial for the normal function of the immune system [9] while constituting a tiny fraction of lymph node cellularity. Expression of the glycoprotein podoplanin (PDPN) and the adhesion molecule CD31 (also known as platelet and endothelial cell adhesion molecule 1 [PECAM-1]) among CD45 ${ }^{-}$cells identifies four LNSC populations: PDPN ${ }^{+} \mathrm{CD} 1^{-}$fibroblastic reticular cells (FRCs), $\mathrm{PDPN}^{+} \mathrm{CD} 31^{+}$lymphatic endothelial cells (LECs), PDPN ${ }^{-} \mathrm{CD} 1^{+}$blood endothelial cells (BECs) and the heterogeneous PDPN CD31 double-negative cells (DNCs). FRCs produce and regulate a specialised reticular network of fibres used by lymphocytes and dendritic cells as 
a scaffold on which to migrate and interact. FRCs also secrete chemokines and survival factors that attract and maintain $\mathrm{T}$ cells $[10,11]$. LECs line lymphatic vessels and facilitate the entry of antigen-bearing dendritic cells and soluble antigens into lymph nodes, while BECs form blood vessels through which naive lymphocytes also gain access to lymph nodes. FRCs and LECs have so far been implicated in tolerance induction [12] via the presentation of endogenously expressed tissue-restricted antigens (TRAs) in the presence of programmed death-ligand 1 (PD-L1) and/or absence of costimulatory molecules $[12,13]$. While the role of LNSCs in organising and regulating immune responses is now established, these cells have not yet been studied in the context of human autoimmune diseases or targeted in immunotherapy. Our knowledge of LNSCs is largely based on mouse data, and whether these can be extrapolated to humans remains to be determined.

Given the extensive evidence of type 1 diabetes-associated alterations in professional APCs [4] and the potential of LNSCs as tolerogenic APCs, we set out to evaluate whether LNSCs are also altered in the PLNs of individuals with type 1 diabetes and NOD mice, relative to individuals without diabetes and control mice, in order to address their possible contribution to loss of tolerance and their putative utility in antigen-specific therapy. To this end, we assessed the relative frequency of LNSC subsets and their expression of functional molecules related to antigen presentation and tolerance induction using FACS and gene expression analysis.

\section{Methods}

Mice Female NOD mice (Jax \#001976) and non-obese diabetes-resistant (NOR) mice (Jax \#002050) were obtained from The Jackson Laboratory (Sacramento, CA, USA and Bar Harbor, ME, USA, respectively) and bred in our animal facility under specific pathogen-free conditions. Only females were analysed because NOD females have a higher incidence of disease relative to males, and we needed to maximise our ability to detect disease-related alterations. Two age groups of mice were used: 4-6 weeks (initiation stage) and 12-14 weeks (advanced prediabetes stage), and were referred to in our study as 'young' and 'old', respectively, not in absolute terms but in relative terms. Most mice in the 'old' group $(\sim 90 \%)$ had not yet developed hyperglycaemia at the time of analysis. Animal protocols were approved by the Institutional Animal Care and Use Committee at Columbia University.

Donors PLN tissues were obtained from unidentified deceased organ donors diagnosed with type 1 diabetes; without diabetes but with type 1 diabetes-associated autoantibodies $\left(\mathrm{AAb}^{+}\right)$; or without diabetes and autoantibody-negative (control donors). All tissues from donors with type 1 diabetes $(n=16$, median age 23 years [range $9-30$ years], $44 \%$ male) and $\mathrm{AAb}^{+}$donors $(n=4$, median age 21 years [range $17-23$ years], 50\% male) were obtained from the Network of Pancreatic Organ Donors with Diabetes (nPOD). Control tissues were from non-diabetic organ donors $(n=30$, median age 36 years [range 6-73 years], $63 \%$ male), and were obtained from nPOD and LiveOnNY, as previously described $[14,15]$ (see electronic supplementary material [ESM] Table 1, ESM Fig. 1a); we found no significant differences between these two sources in our results. There was no randomisation or blinding; samples from donors were accepted as they became available and the type of sample was known by the investigator. Unless otherwise specified, $\mathrm{AAb}^{+}$donors were grouped with the type 1 diabetes donors in data analysis on the basis that they all had islet AAb (underlying islet autoimmunity). The use of human tissues from deceased organ donors does not qualify as human subjects as determined by the Institutional Review Board of the Human Research Protection Office at Columbia University.

Stromal cell isolation PLNs from human donors were trimmed of surrounding fat, cut into small pieces and digested for $30 \mathrm{~min}$ at $37^{\circ} \mathrm{C}$ with gentle stirring in DMEM containing collagenase IV ( $1 \mathrm{mg} / \mathrm{ml}$; Worthington, Lakewood, NJ, USA), dispase ( $1 \mathrm{mg} / \mathrm{ml}$; Invitrogen, Darmstadt, Germany), DNase I (100 $\mu \mathrm{g} / \mathrm{ml}$; Roche, Basel, Switzerland), $\mathrm{CaCl}_{2}$ ( $3 \mathrm{mmol} / \mathrm{l})$ and $2 \% \mathrm{FCS}$. Released cells were filtered, washed twice and resuspended in DMEM with 10\% FCS.

Because of the paucity of LNSCs and the small size of mouse PLNs, four to five mice were pooled for each replicate. PLNs collected from female NOD and NOR mice were cut into small pieces and digested with Liberase $(0.5 \mathrm{mg} / \mathrm{ml}$; Roche) for $30 \mathrm{~min}$ at $37^{\circ} \mathrm{C}$. Stromal cells (mouse and human) were enriched after depletion of lymphocytes and contaminating erythrocytes using biotinylated anti-CD3, CD19 and CD235a (for human PLNs) and anti-CD45 and TER-119 (for mouse PLNs) (ESM Table 2) through magnetic bead isolation (EasySep Anti-Biotin Kit; StemCell, Vancouver, BC, USA), or MojoSort Streptavidin Kit (BioLegend, San Diego, CA, USA).

Stromal cell analysis and sorting We identified the four LNSC populations among $\mathrm{CD} 45^{-}$cells using the aforementioned PDPN and CD31 markers, as well as HLA-DR ${ }^{\text {high }} \mathrm{CD} 45^{\text {low }}$ cells among $\mathrm{CD} 45^{+}$cells. The latter were also of interest as: (1) they had been included in previous LNSC studies because of their lower expression of CD45 and radioresistance; and (2) they are enriched in autoimmune regulator (AIRE) ${ }^{+}$dendritic cells $[16,17]$. In addition, human cells were stained for HLADR (MHC-II), HLA-A,B,C (MHC-I) and PD-L1, and mouse cells for I-A ${ }^{\mathrm{g} 7}$ (MHC-II; detected with the cross-reacting anti$\mathrm{I}^{-\mathrm{A}^{\mathrm{k}}}$ antibody), H2-K ${ }^{\mathrm{d}}$ (MHC-I) and PD-L1 (ESM Table 2). After staining, cells were acquired using a Fortessa cell analyser (BD, Franklin Lakes, NJ, USA) and FCS Express 6 
(DeNovo Software, Glendale, CA, USA) was used for data analysis. Mean fluorescence intensity (MFI) values were normalised in each sample against $\mathrm{CD} 45^{+} \mathrm{MHC}-\mathrm{II}^{-}$cells as control and represent a fold change. If the number of cells was sufficient, cell populations were sorted into TRI Reagent LS (Sigma, St Louis, MO, USA) using the BD Influx cell sorter and stored at $-80^{\circ} \mathrm{C}$.

Targeted analysis of gene expression in LNSCs from human PLNs using Biomark Total RNA was isolated from cells sorted in TRI Reagent LS, using a modified protocol of chloroform extraction followed by purification using an RNeasy Micro Kit (Qiagen, Hilden, Germany). cDNA was synthesised from $200 \mathrm{ng}$ of total RNA for each sample using iScript Reverse Transcription Supermix (Bio-Rad, Hercules, CA, USA). All Delta Gene primers (Fluidigm, South San Francisco, CA, USA) were purchased pre-validated for assay performance (ESM Table 3). Samples were pre-amplified using a pool of all primers (minus endogenous controls; $50 \mathrm{nmol} / \mathrm{l}$ final) and 18 cycles, as per the Biomark protocol. Samples (16 in triplicates) were loaded onto Biomark $48 \times 48$ IFC chips (Fluidigm) and assayed against the 48 Eva Green-based assays (primers at $5 \mu \mathrm{mol} / \mathrm{l}$ final) listed in ESM Table 4. Target gene expression was calculated using the comparative method for relative quantification after normalisation to expression of the housekeeping HPRT1 gene, the expression of which was the most homogeneous across multiple samples compared with other housekeeping genes. An insufficient number of sorted cells, poor RNA quality (assessed using BioAnalyzer PicoChip; Agilent Technology, Waldbronn, Germany) or failed amplification were criteria for sample exclusion in the gene expression analysis. For comparison of relative gene expression between human and mouse LNSC subsets (our data vs Immunological Genome Project [ImmGen] RNASeq data [www.immgen.org]), we normalised gene expression to $100 \%$ in subsets in which it was most highly expressed in each set of data independently. Biomark data were deposited at: https://ata.mendeley.com/datasets/ d9rdzdmvyf/1 [18].

Statistical and R analysis Statistical testing was performed using GraphPad Prism 5.0 (San Diego, CA, USA). Unless otherwise indicated, values are expressed as means \pm SEM. Principal component analysis and t-distributed stochastic neighbour embedding unsupervised clustering plots were generated using R 3.5.1 software (R Core Team 2018, Boston, MA, USA) and the Singular Analysis Toolset package from Fluidigm (v.3.6.2; www. fluidigm.com/software). Statistical significance of differences between the groups were analysed using twotailed Student's $t$ tests, and the $p$ values are reported in the figures. Additional statistical tests are described in the figure legends.

\section{Results}

Altered distribution of LNSC subsets in PLNs Subsets of LNSCs were defined by gating CD45 ${ }^{-}$cells after tissue digestion and cell enrichment (Fig. 1a). We assessed the relative frequency of the three most homogeneous LNSC subsets (FRCs, LECs and BECs), as DNCs represent a heterogeneous population that is variably contaminated by erythrocytes, despite efforts to remove them. This may confound frequency results without affecting gene expression results. PLNs from donors with type 1 diabetes had relatively fewer FRCs and more BECs compared with PLNs from control donors (Fig. 1b). These altered proportions came predominantly from female donors (Fig. 1c), although similar (yet not significant) changes were observed in male donors (Fig. 1d). Differences in subset distribution were not due to age and, although the FRC frequency significantly decreased with age (ESM Fig. 1b), donors with type 1 diabetes were younger than control donors on average, making the type 1 diabetes-associated reduction in FRCs even more meaningful. Interestingly, similar alterations in FRC and BEC frequency observed in human PLNs were also seen in PLNs of NOD female mice compared with control NOR female mice, analysed at different ages (Fig. 1e,f). While BECs predominated in human lymph nodes, FRCs were proportionally more abundant than LECs or BECs in mice. We did not find differences between PLNs and other lymph nodes in the distribution of human LNSC subsets (ESM Fig. 1f). PDPN expression was significantly higher on LECs than FRCs, but its expression was non-significantly reduced $(p=0.06)$ on LECs from donors with type 1 diabetes relative to control or $\mathrm{AAb}^{+}$donors (Fig. 1g-i). PDPN expression on LECs was also lower in NOD vs NOR mice, and this was already evident in young animals (Fig. 1j).

Increased expression of MHC-II and PD-L1 in LNSCs from human PLNs HLA-DR (MHC-II) is typically expressed at lower levels in LNSCs compared with dendritic cells or other haematopoietic APCs, with BECs expressing higher levels than LECs and FRCs (Fig. 2a). We found no significant agerelated changes in HLA-DR expression in our control donors, with the exception of FRCs, in which HLA-DR increased with age (ESM Fig. 2a-c). However, the expression of HLA-DR was highly upregulated across all LNSC subsets in PLNs from type 1 diabetes donors (Fig. 2b-d), and this was seen equally in both sexes, regardless of age (ESM Fig. 2a-e). PD-L1 was also mainly expressed on endothelial cells, and was significantly upregulated in BECs from the PLNs of type 1 diabetes donors (Fig. 2e-g). When $\mathrm{AAb}^{+}$donors were considered separately, the increased frequency of $\mathrm{HLA}_{-}-\mathrm{DR}^{+}$was already evident in all LNSC subsets from $\mathrm{AAb}^{+}$donors, and the frequency of PD-L1 ${ }^{+}$cells was increased in BECs (ESM Fig. $2 \mathrm{f}, \mathrm{g}$ ). In contrast, increases in expression levels (MFI) of HLA-DR in all LNSC subsets and PD-L1 in DNCs were 
Fig. 1 Stromal cell distribution in human and mouse PLNs. (a) Representative dot plots of PDPN and CD31 expression gated on $\mathrm{CD} 45^{-}$cells from control (blue) and type 1 diabetes (red) PLN samples. (b-d) Relative subset distribution among FRCs, LECs and BECs between human control and type 1 diabetes in samples from (b) all donors, (c) female donors and (d) male donors. (e) Relative distribution among FRCs, LECs and BECs in PLNs from NOR and NOD mice from two different age groups. (f) Representative dot plots of PDPN and CD31 expression gated on $\mathrm{CD} 45^{-}$cells from $\mathrm{NOR}$ (blue) and NOD (red) PLNs. $(\mathbf{g}, \mathbf{h})$ Normalised PDPN MFI expression in FRCs and LECs from $(\mathbf{g})$ control $(n=20)$ vs type 1 diabetes (with $\mathrm{AAb}^{+}, n=14$ ) samples and (h) control $(n=20)$ vs $\mathrm{AAb}^{+}(n=4)$ or type 1 diabetes samples $(n=20)$. Reduction in PDPN on LECs between control and type 1 diabetes did not reach significance $(p=0.06)$. (i) Representative histograms of PDPN expression in FRCs and LECs in PLN tissue from donors with and without type 1 diabetes. Grey histograms represent DNCs. (j) Normalised PDPN MFI expression in FRCs and LECs in PLNs from NOR $(n=6)$ and NOD $(n=10)$ mice from two different age groups. $* p<0.05$, $* * p<0.01, * * * p<0.001$, by twotailed Student's $t$ test. T1D, type 1 diabetes a
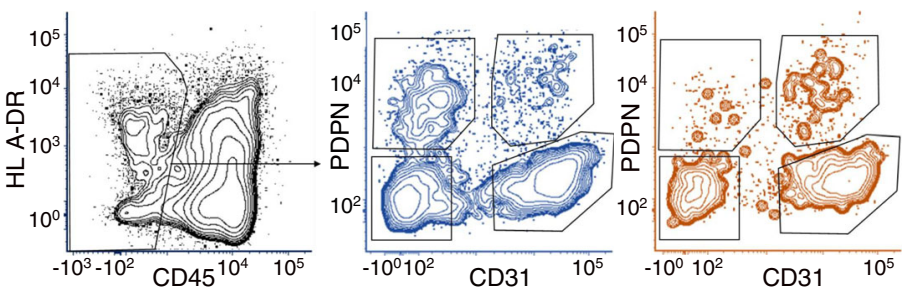

b

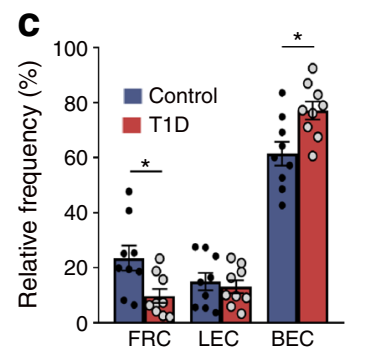

d

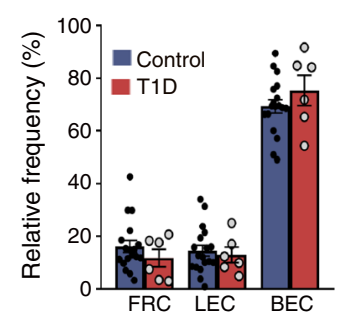

e

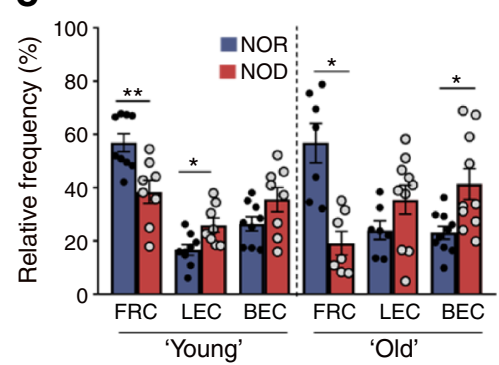

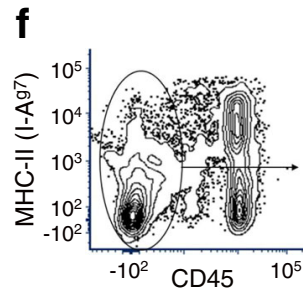
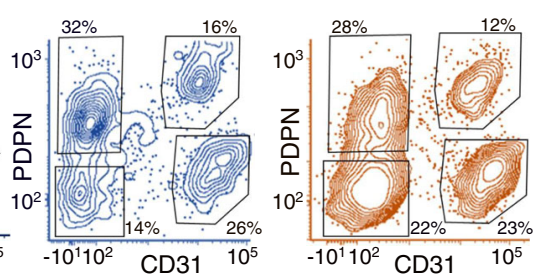

i

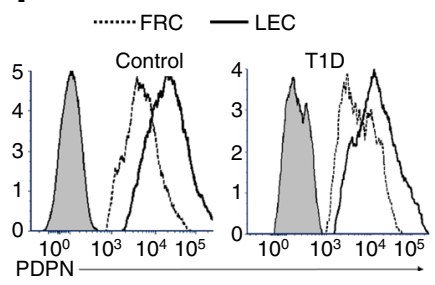

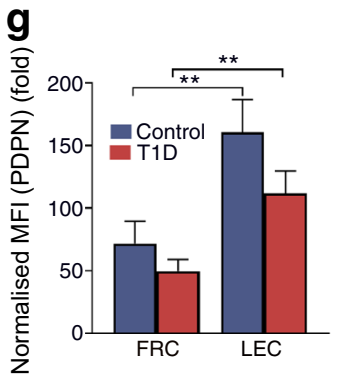
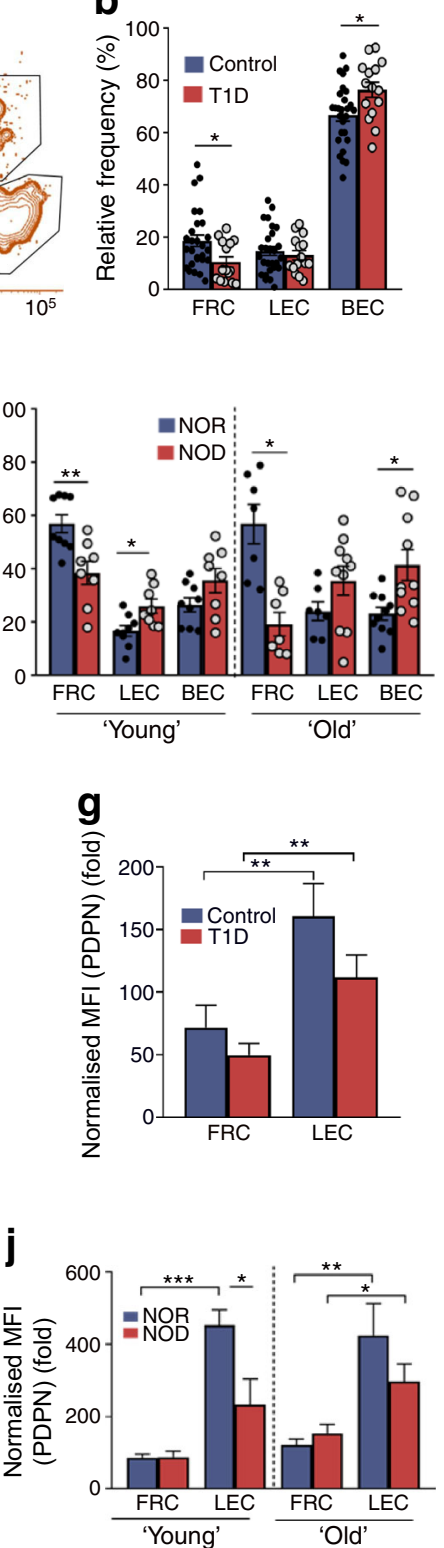

mostly evident after diagnosis (Fig. 2h,i). In mice, MHC-II expression was not increased in PLNs of NOD vs NOR mice (ESM Fig. 2h). While PD-L1 expression was mostly restricted to endothelial cells, the low expression in fibroblastic cells was upregulated in NOD mice (ESM Fig. 2i). No significant differences were found in the expression of MHC-I in either humans (Fig. 2j) or mice (ESM Fig. 2j,k).

\section{Human LNSCs share basic tolerogenic features with murine} LNSCs We explored the expression of 44 TRAs and tolerancerelated genes (ESM Table 4) using normalised human LNSC data obtained from the high-throughput Biomark HD quantitative PCR platform. In unsupervised principal component analysis or t-distributed stochastic neighbour embedding cluster analysis of the four subsets of LNSCs, fibroblastic cells
(FRCs and DNCs) clustered separately from endothelial cells (LECs and BECs) (Fig. 3a,b). Moreover, LNSC clusters (particularly FRCs and DNCs) from type 1 diabetes donors generally segregated from their counterparts from control samples (Fig. 3b), demonstrating the influence of disease on their gene expression profile. We next compared our normalised human LNSC gene expression data with the RNA-Seq gene expression profile of mouse LNSCs, publicly available from the Immunological Genome Project (ImmGen database) (Fig. $3 c, d)$. Overall, the expression patterns of different genes were similar between humans and mice, with certain genes restricted to endothelial (LEC/BEC) or fibroblastic (FRC/DNC) cells in general (e.g. LGALS9, CD274 [encoding PD-L1] and NOS2 in endothelial cells; LGALS1, FAS, ICOSLG and $A L D H 1 A 1$ in fibroblastic cells), or restricted to specific 
Fig. 2 MHC-II, PD-L1 and MHC-I expression in LNSCs from human PLNs. (a)

Representative dot plot of HLADR and CD31 expression gated on $\mathrm{CD} 45^{-}$cells from a control human PLN, showing overlay of the four LNSC subsets. (b) Percentage of HLA-DR ${ }^{+}$LNSCs comparing control (blue bars)vs type 1 diabetes (red bars) donors. (c) Histograms of HLA-DR expression on the four LNSC subsets from control and type 1 diabetes donors. (d) Normalised HLA-DR MFI comparing control $(n=20)$ vs type 1 diabetes $(n=$

14) donors. (e) Histograms of PDL1 expression on LNSC subsets from control and type 1 diabetes donors and representative dot plots of PD-L1 expression on $\mathrm{CD} 31^{-}$and $\mathrm{CD} 31^{+}$cells, overlaying the four subsets of LNSCs from control and type 1 diabetes donors. (f, g) Percentage of (f) $\mathrm{PD}-\mathrm{L1}^{+}$and (g) normalised PD-L1 MFI on LNSCs from control and type 1 diabetes donors. In (g): control, $n=8$; type 1 diabetes, $n=10$. (h, i) Normalised (h) HLA-DR MFI and (i) PD-L1 MFI comparing control $(n=8), \mathrm{AAb}^{+}(n=4)$ and type 1 diabetes $(n=10)$ donors. (j) Normalised HLA-A,B,C MFI on LNSC subsets from control $(n=5)$ and type 1 diabetes $(n=9)$ donors. $* p<0.05$, ** $p<0.01$, $* * * p<0.001$, by two-tailed Student's $t$ test; ${ }^{\dagger} p<0.05$, by ANOVA with multiple comparisons test. Additional statistics: (f) FRC, $p=0.08$; (g) $\mathrm{DNC}, p=0.07$; (i) FRC, $p=0.07$. T1D, type 1 diabetes
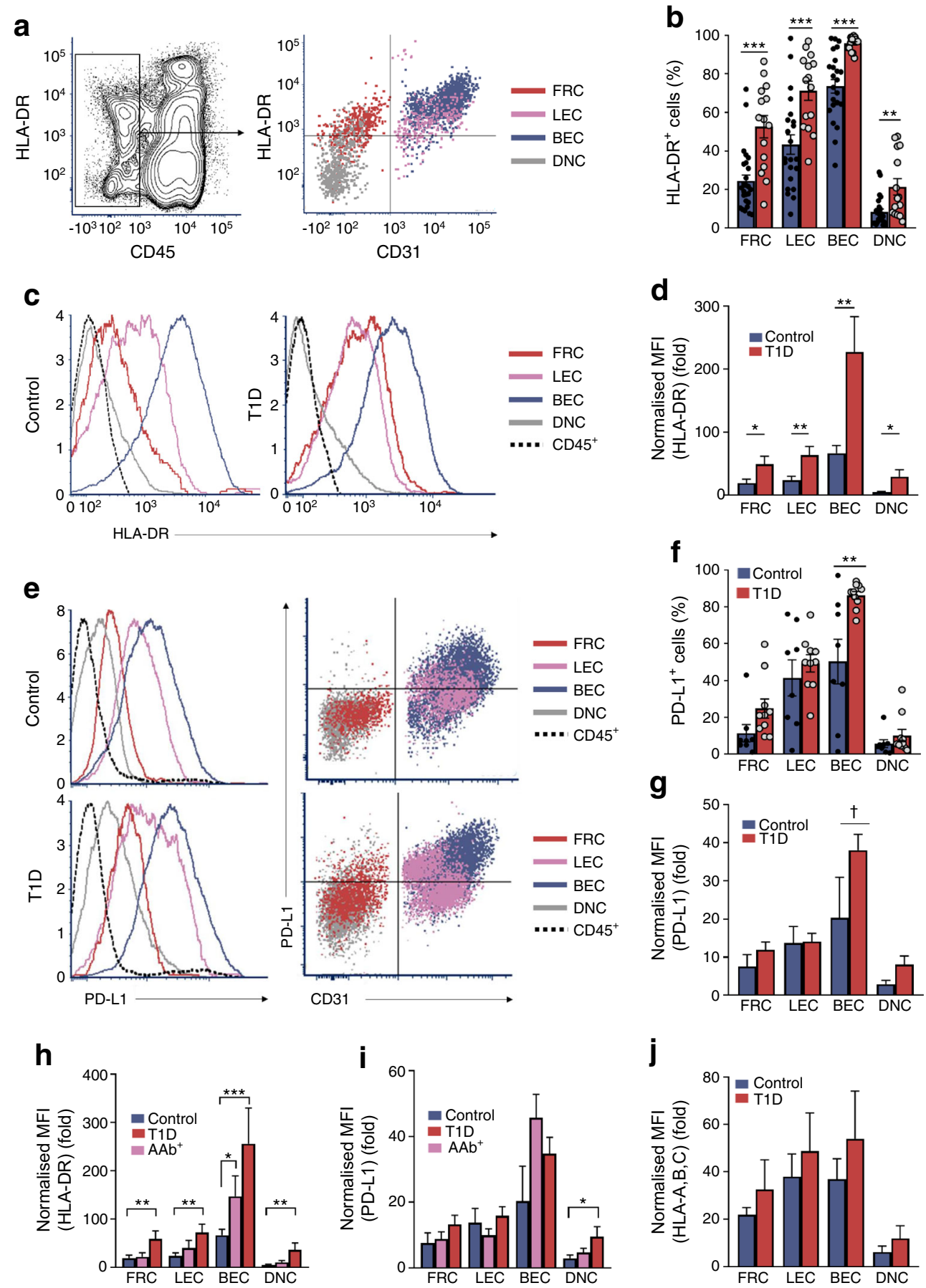

LNSC subsets (e.g. TSLP in FRCs or ENTPD1 in BECs). TRAs were weakly expressed and not consistently detected across samples, leading to more variability (ESM Fig. 3a, ESM Table 5). Low gene expression for some of the markers, particularly TRAs, in LNSCs became evident when compared with their expression in sorted HLA-DR ${ }^{\text {high }} \mathrm{CD} 45^{\text {low }}$ cells (AIRE-enriched dendritic cells; ESM Fig. 3b).

The tolerance-related gene expression profile of LNSCs in PLNs is reinforced in type 1 diabetes Further analysis of the transcription profile of LNSCs provided new insights on their functional capabilities as tolerogenic APCs and how these might be affected in type 1 diabetes. Overall, between all LNSC subsets, FRCs seem to differ the most between type 1 diabetes and control samples, as revealed by cluster analysis (Fig. 3b), although a number of genes were also affected in type 1 diabetes in other LNSC subsets (ESM Table 5). We first assessed to what extent the expression of TRAs and transcription factors that might regulate them was altered in the context of type 1 diabetes. Genes encoding the transcription factors 

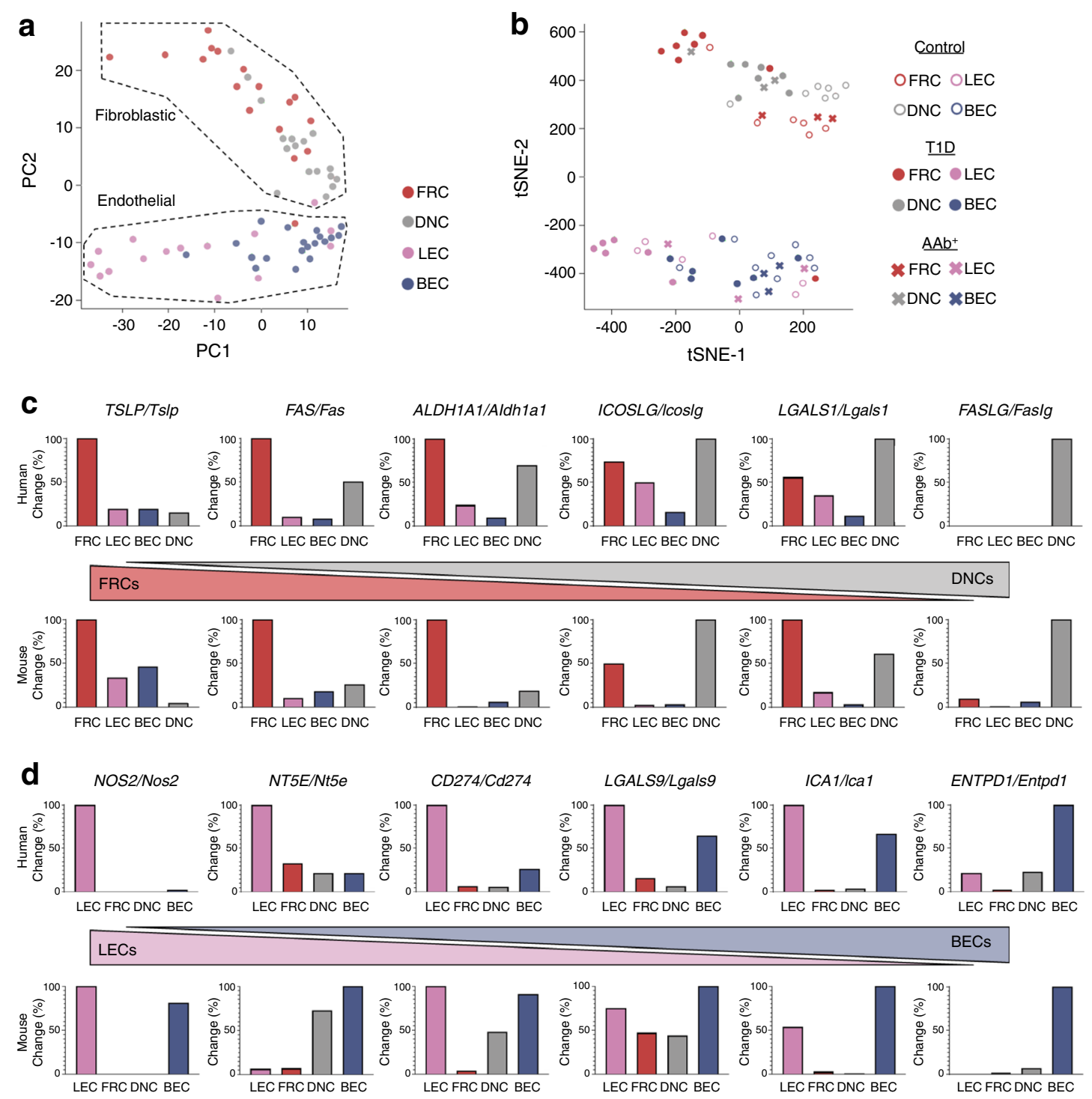

Fig. 3 Tolerogenic gene expression profile in human and mouse LNSCs. (a) Principal component analysis and (b) t-distributed stochastic neighbour embedding (tSNE) analysis of the four LNSC subsets in human PLNs from control, $\mathrm{AAb}^{+}$and type 1 diabetes donors. (c, d) Comparison of relative expression of genes preferentially expressed in

(c) fibroblastic cells or (d) endothelial cells, comparing human (control donors, $n=5$ ) and mice (C57BL/6, $n=3$ ) for the four subsets of LNSCs from our data and the ImmGen database, respectively. PC, principal component; T1D, type 1 diabetes

AIRE and FEZ family zinc finger 2 (FEZF2), first described in medullary thymic epithelial cells, were barely detected in human LNSCs (ESM Fig. 3). DEAF1, which has homologies with AIRE, was expressed in human LNSCs but no differences were found between type 1 diabetes and control samples (Fig. 4a). Most TRAs, related or not to beta cells, were barely detected in LNSCs compared with dendritic cells (ESM Fig. 3b), but some were either significantly upregulated (e.g. ICA1, and MLANA; $p<0.05$ ) or more likely to be detected (e.g. INS and TYR; $p>0.05$ ) in specific LNSC subsets in type 1 diabetes samples vs control samples (Fig. 4b-e). Among the genes analysed (the function of which are described in ESM Table 4), ALDH1A1, TNFRSF14, ICOSLG, IL12A (encoding IL-35 $\alpha$ ), NT5E, LGALS1 and PDCD1LG2 (encoding PD-L2) were upregulated in FRCs from type 1 diabetes donors, with some of these changes reaching significance (Fig. 4f-j, ESM Fig. 4a,h). A substantial number of genes in LECs from type 1 diabetes donors had increased expression, including TNFRSF14, ENTPD1, TGFB1, LGALS9, EBI3 (encoding IL-35ß), NOS2, ARG2 and PDCD1LG2, but only the change in ENTPD1 was significant (Fig. 4g,k-p and ESM Fig. 4h). Finally, type 1 diabetes-associated upregulation was seen for $A L D H 1 A 1, A R G 2$ and FAS in DNCs (Fig. 4f,p and ESM Fig. $4 \mathrm{e})$.

Defective immunosuppressive potential of human HLA-DR ${ }^{\text {high }}$ CD45 ${ }^{\text {low }}$ cells in type 1 diabetes In the thymus, the expression of many TRAs in medullary thymic epithelial cells is 
Fig. 4 Tolerogenic gene expression profile in LNSCs from control vs type 1 diabetes PLNs. Relative mRNA expression of selected genes (analysed by quantitative PCR) in human LNSCs comparing control (black), $\mathrm{AAb}^{+}$(grey) and type 1 diabetes (white) donors.

Representative genes shown correspond to transcription factors, enzymes, secreted immunoregulatory molecules and inhibitory cell surface ligands, as indicated in ESM Table 4. (a) $D E A F 1$, (b) INS, (c) ICAI, (d) TYR, (e) MLANA, (f) ALDHIAl, (g) TNFRSF14, (h) ICOSLG, (i) IL12A, (j) NT5E, (k) ENTPD1, (l) $T G F B 1$, (m) $L G A L S 9$, (n) EBI3, (o) NOS2 and (p) ARG2. All mRNA levels were normalised against endogenous control HPRT1. ${ }^{*} p<0.05$, by two-tailed Student's $t$ test. B, BEC; D, DNC; F, FRC; L, LEC
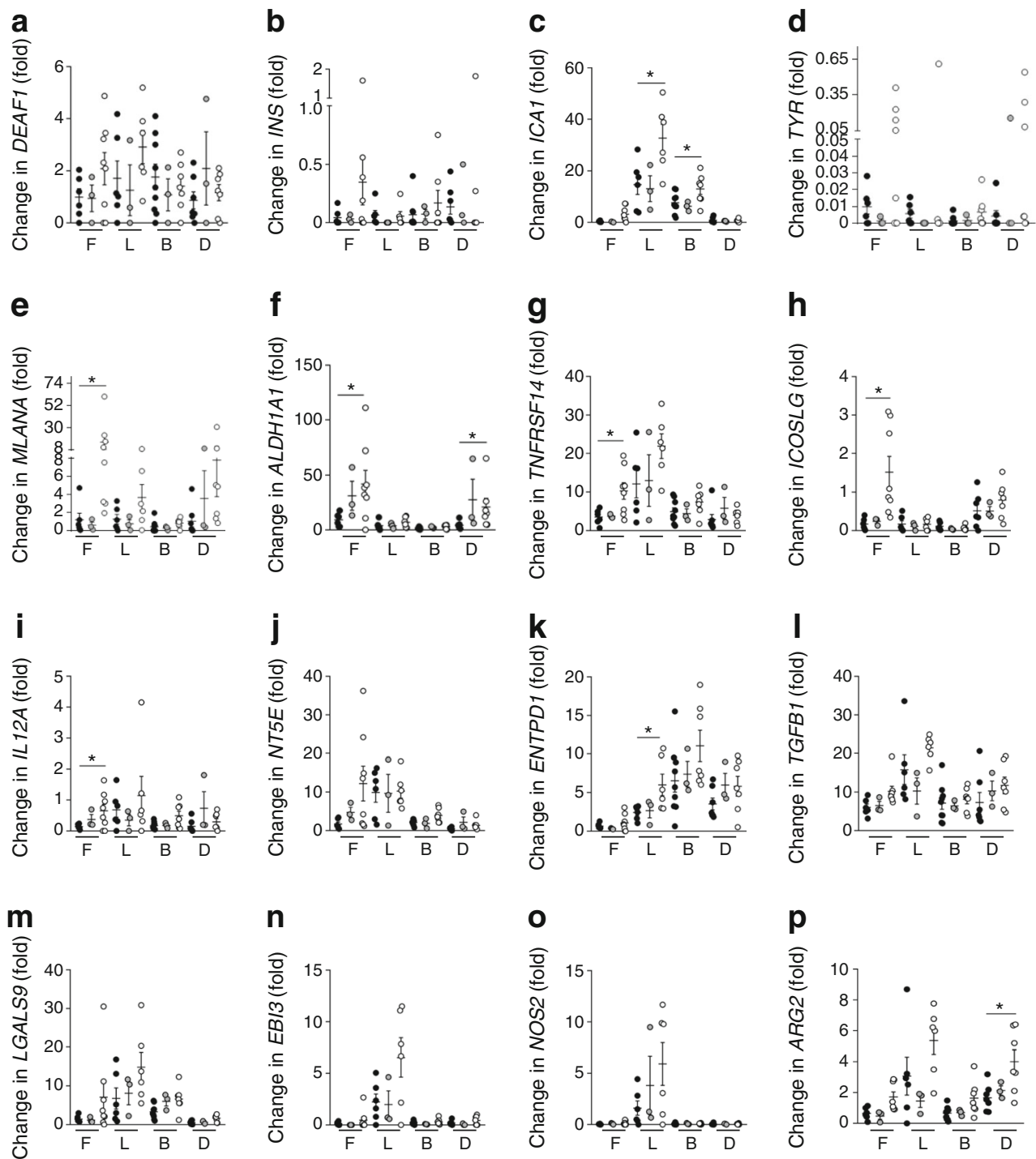

regulated by AIRE and/or FEZF2, and allows for the deletion of specific autoreactive $\mathrm{T}$ cells and/or positive selection of regulatory $\mathrm{T}$ cells. In lymph nodes, AIRE expression marks a subset of dendritic cells with potent tolerogenic properties $[17,19,20]$. These peripheral $\mathrm{AIRE}^{+}$dendritic cells, originally thought to be stromal cells, are found among a discrete population of HLA-DR ${ }^{\text {high }} \mathrm{CD} 45^{\text {low }}$ cells, which we analysed in human PLNs and found to be diminished in type 1 diabetes vs control donors (Fig. 5a,b). The level of AIRE mRNA expression in that population was not significantly different in type 1 diabetes vs control donors (Fig. 5c). No major differences were seen in the expression of different TRAs, with the exception of ICAI $(p=0.08)$ and INS $(p<0.05)$, for which over $80 \%$ of type 1 diabetes donors had higher expression than control donors (Fig. 5d). In contrast to LNSCs, there was more downregulation of tolerance-related genes than upregulation in these cells (ESM Table 5), with FAS and IDOI (both $p<0.05$ ) and $E B I 3$ (beta chain for IL-27 and IL-35; $p=$
0.07 ) being the most reduced (Fig. 5e-g). Thus, the tolerogenic capabilities of these cells may be hindered in type 1 diabetes.

\section{Discussion}

In the current study, we characterised human LNSC populations (as well as AIRE-enriched dendritic cells) in PLNs from control vs type 1 diabetes donors in terms of phenotype and gene expression profile of several tolerance-related genes and TRAs, providing a unique opportunity to identify potential alterations in these populations associated with type 1 diabetes. Among the four LNSC subsets, the two fibroblastic subsets (FRCs and DNCs) shared more features between themselves than with the two endothelial subsets (LECs and BECs). Many of these features for each subset were also comparable between humans and mice. Of particular interest was 
Fig. 5 Type 1 diabetes-associated defects in HLA-DR ${ }^{\text {high }} \mathrm{CD} 45^{\text {low }}$ cells in PLNs. (a) Representative dot plots showing the HLA$\mathrm{DR}^{\text {high }} \mathrm{CD} 45^{\text {low }}$ cell population studied that contains AIREenriched dendritic cells. (b) Percentage of HLA-DR ${ }^{\text {high }}$ $\mathrm{CD} 45^{\text {low }}$ cells, gated on live singlets, in PLNs from control, $\mathrm{AAb}^{+}$and type 1 diabetes donors. (c) Relative AIRE mRNA expression in sorted LNSCs and HLA-DR ${ }^{\text {high }}$ CD $45^{\text {low }}$ cells from control (blue) and type 1 diabetes (red) PLNs. (d-g) Relative mRNA expression (analysed by quantitative PCR) in sorted HLA$\mathrm{DR}^{\text {high }} \mathrm{CD} 45^{\text {low }}$ cells between control (blue bars; $\mathrm{AAb}^{-}$, black circles; $\mathrm{AAb}^{+}$, pink circles) and type 1 diabetes (red bars with grey circles) donors of selected genes corresponding to (d) TRAs, (e) immunosuppressive enzymes, (f) secreted immunoregulatory molecules and (g) inhibitory cell surface ligands. All mRNA levels are normalised against endogenous control HPRT1. * $p<$ 0.05 , by two-tailed Student's $t$ test. For some genes, significance was not reached due to a single outlier (e.g. $I C A 1, p=0.08 ; E B I 3$, $p=0.07)$. T1D, type 1 diabetes
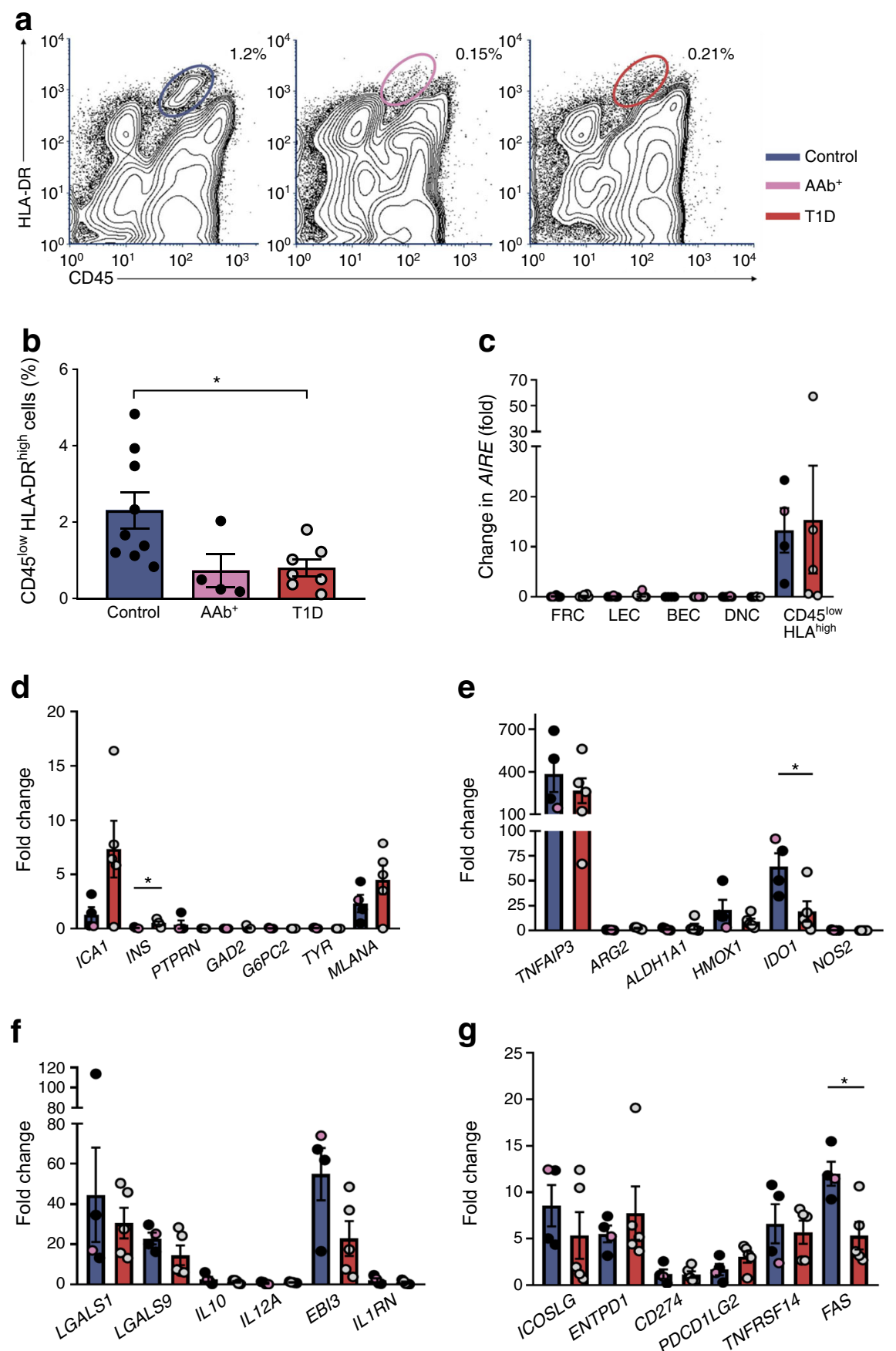

the similarity in altered distribution of the different LNSC subsets in PLNs between humans and mice in the context of type 1 diabetes. More specifically, FRCs were decreased in proportion, while BECs were increased (particularly in women). These alterations were disease-dependent and not agedependent, although we did observe a significant decrease over time for human FRCs, in line with a prior report using old mice (14-26 months) [21]. Further studies, including analysis of PLN sections by immunofluorescence, will help us to understand if type 1 diabetes is also associated with structural changes in PLNs. A reduction in the FRC population might also indicate specific killing by immune cells (e.g. if expressing insulin, as shown in mice [22-24], or receiving MHC/ peptide complexes from islet-derived dendritic cells [25]), and the increased expression of PD-L1 might indicate an attempt to fend off this assault.

Overall, analysis of human LNSCs derived from the PLNs of type 1 diabetes donors revealed that these cells, far from being defective in their tolerogenic capabilities, in fact overexpress many of the tolerance-related genes analysed in this 
study. In particular, the fibroblastic subsets had the most type 1 diabetes-associated gene expression alterations among LNSCs (13 genes [46\%] and 12 genes [43\%] upregulated with more than a twofold change in FRCs and DNCs, respectively) (ESM Table 5). Conversely, in the AIRE-enriched (HLA$\mathrm{DR}^{\text {high }} \mathrm{CD} 45^{\text {low }}$ ) dendritic cell population, there were twice as many downregulated as upregulated genes among this list in type 1 diabetes vs control samples (six downregulated, three upregulated), including significant downregulation of IDO1 and FAS (ESM Table 5).

TRA expression by LNSCs may impact some autoreactive T cells. LNSCs that express self-antigens can delete autoreactive $\mathrm{CD}^{+} \mathrm{T}$ cells $[22,26-28]$, and ectopic expression of INS was reduced in whole PLNs from type 1 diabetes donors [29]. When we analysed specific LNSC subsets, we found no change in the expression of INS; however, genes encoding other TRAs such as ICA1 and MLANA were increased in LECs and FRCs, respectively. Likewise, $D E A F 1$ expression was not changed overall, consistent with our previous report that $D E A F 1$ is affected at the level of mRNA splicing and not at the transcriptional level [30]. Thus, aberrant autoreactive T cell responses in human type 1 diabetes may not be due to a lack of TRA expression by LNSCs, although the very low expression levels of islet TRAs may limit presentation of endogenously expressed TRAs by LNSCs in PLNs relative to the presentation of islet-derived antigens by dendritic cells. Whether human LNSCs do interact with diabetogenic $\mathrm{T}$ cells in an antigen-dependent manner remains unclear.

Similar to mice $[28,30]$, inflammation can trigger phenotypic and transcriptional changes in human LNSCs [31], and lipopolysaccharide upregulates HLA-DR and PD-L1 in human FRCs [32]. PD-L1 is also upregulated by type I IFN during inflammation [33], and it is actually one of the mechanisms used by LNSCs (at least LECs) to control autoreactive T cells in mice [13]. Similarly, PD-L1 is upregulated in beta cells from humans with type 1 diabetes and NOD mice, in association with T cell infiltration and IFN $\gamma$ production [34]. MHC-II expression on mouse LNSCs is low yet appears to be sufficient to affect autoreactive $\mathrm{CD}^{+} \mathrm{T}$ cells and contribute to the maintenance of regulatory $\mathrm{T}$ cells [35], and lack of MHC-II expression by LNSCs can result in autoimmunity in mice [36]. HLA-DR expression on human LNSCs is higher than in mice and, in the context of type 1 diabetes, was highly upregulated, along with PD-L1 to some extent. It is likely that many of these observed LNSC alterations are induced by inflammation (i.e. a consequence of the disease), where IFN $\gamma$ produced by diabetogenic $\mathrm{T}$ helper type 1 cells might play a key role. If inflammation is a driving factor in the upregulation of MHC-II in type 1 diabetes, it is possible that the reason we did not observe increased MHC-II in NOD mice is that, at 12-14 weeks of age, the mice were not old enough or not at a sufficiently advanced stage of disease. In addition to loading of endogenously expressed self-antigens on MHC, LNSCs may also acquire MHC/peptide complexes during interactions with migratory dendritic cells and use them to induce deletion and/or anergy [25]. The ability of LNSCs to capture and process exogenous antigens for presentation is not established as they are expected to be outcompeted by professional APCs, which are more efficient in this role [12]. As we only measured the expression of HLA-DR on the surface of LNSCs, we cannot establish how much of it comes from intrinsic expression and how much from transfer from other APCs. However, expression of PDPN is important for the interaction of dendritic cells with FRCs/LECs [37], and since PDPN expression was downregulated in individuals with type 1 diabetes (at least in LECs), it may be expected that FRC-dendritic cell interactions, and therefore transfer of HLA-DR from dendritic cells, would be reduced rather than increased, suggesting that the increased HLA-DR expression may rather be a consequence of inflammation. Some of the reported alterations (e.g. relative LNSC frequencies, PDPN on LECs) occurred early (evident in 'young' NOD mice) and may contribute to disease progression. Early alterations might also be influenced by genetic factors; thus, studies comparing mouse strains and inflammatory conditions are needed for more mechanistic insights.

The expression of ALDH1A1 (required for retinoic acid synthesis) and LGALSI (encoding the anti-proliferative galectin-1) in FRCs and their upregulation in type 1 diabetes is interesting, as they are also both upregulated with IFN $\gamma$ and lipopolysaccharide in vitro [32]. Other genes encoding immunomodulatory molecules altered in FRCs from type 1 diabetes donors were ICOSLG, TNFRSF14 and NT5E. This overexpression, together with higher expression of HLA-DR and PD-L1, could indicate a countervailing response to the proinflammatory environment occurring in PLNs in the context of type 1 diabetes, but whether LNSCs can effectively engage autoreactive $\mathrm{T}$ cells to transmit these tolerogenic signals remains to be established.

Collectively, our findings show that LNSCs isolated from the PLNs of type 1 diabetes donors differ from those of control donors in their relative frequency and phenotype, including upregulation of HLA-DR and PD-L1 and several intrinsic abnormalities in their mRNA expression. LNSCs from individuals with type 1 diabetes, particularly fibroblastic cells (FRCs and DNCs), generally overexpress genes related to tolerance, whereas HLA-DR ${ }^{\text {high }} \mathrm{CD} 45^{\text {low }}$ cells, including AIRE-enriched dendritic cells, seem to be defective in a number of tolerogenic pathways.

To our knowledge, this is the first report on type 1 diabetesassociated phenotypic and transcriptional alterations affecting human LNSCs isolated from islet-draining PLNs, and it presents these populations as heterogeneous, responsive to their microenvironment and potential players in balancing immune responses between tolerance and immunogenicity. Our study sheds light on yet unexplored populations of cells that likely influence or are affected by local autoimmune responses. We have provided a first look at specific genes and pathways that 
each LNSC population has at its disposal to potentially promote tolerance and that are affected in type 1 diabetes. While we have only shown one dendritic cell population in this study, to put some of the LNSC data into perspective, we have found that this dendritic cell population exhibits a reduced tolerogenic potential in type 1 diabetes, yet may be capturing and presenting islet antigens, with potentially undesirable consequences. In contrast, LNSCs appear to have an increased tolerogenic potential as well as enhanced antigen presentation capacity, but express no or few relevant antigens and probably have a poor capacity to acquire exogenous islet antigens compared with other professional APCs. Although we cannot draw direct functional implications for LNSCs in type 1 diabetes progression (functional studies are limited by the paucity of LNSCs), these cells reveal an interesting phenotype for peripheral tolerance and they can be manipulated in vivo to express antigens and induce tolerogenic $\mathrm{T}$ cell responses [38]. Thus, we propose that LNSCs may be pertinent targets of antigen-specific therapies.

Acknowledgements The authors thank A. Pugliese (University of Miami) for critical reading of the manuscript; surgeons N. Matsuoka, D. J. Carpenter and T. Senda (Department of Surgery, Columbia University, New York, NY, USA) who collected tissues procured by LiveOnNY; the nPOD Pathology team; and the organ donors and their families who contributed via nPOD and LiveOnNY. We also thank T. Brusko and $\mathrm{H}$. Seay (University of Florida) for providing residual PLN tissue that helped us to set up and optimise our initial studies.

Data availability All data generated or analysed during this study are included in the published article (and its online supplementary files). Biomark gene expression data were deposited on the Mendeley repository at https://data.mendeley.com/datasets/d9rdzdmvyf/1. Any other raw datasets are available from the corresponding author on reasonable request. No applicable resources were generated or analysed during the current study.

Funding These studies were funded by a pilot grant from Columbia's Clinical and Translational Science Award (UL1TR000040), by a JDRF Transition Award (10-2010-790) and by the Helmsley Charitable Trust George S. Eisenbarth nPOD Award for Team Science (2015PGT1D052). JP-F was supported by a Berrie Fellowship in Diabetes Research from the Berrie Foundation and by a postdoctoral fellowship from the ADA (1-18-PDF-151). The research reported in this publication was supported by nPOD (RRID: SCR 014641), a collaborative type 1 diabetes research project sponsored by the JDRF (nPOD: 5-SRA-2018557-Q-R) and The Leona M. \& Harry B. Helmsley Charitable Trust (Grant no. 2018PG-T1D053). Organ Procurement Organizations partnering with nPOD to provide research resources are listed at www. jdrfnpod.org//for-partners/npod-partners. Studies were also performed using the CCTI Flow Cytometry Core, supported in part by the Office of the Director, National Institutes of Health (awards S10RR027050 and S10OD020056), and by the Diabetes Research Center (grant P30DK063608).

Duality of interest The authors declare that there is no duality of interest associated with this manuscript.

Contribution statement JP-F and RJC designed the experiments. JP-F performed the experiments and analysed the data. DLF contributed lymph node samples via LiveOnNY and experimental design insights. RJC directed the research. JP-F and RJC wrote the manuscript. All authors critically edited and approved the manuscript. RJC is the guarantor of this work and, as such, had full access to all the data in the study and takes responsibility for the integrity of the data and the accuracy of the data analysis.

\section{References}

1. Katsarou A, Gudbjornsdottir S, Rawshani A et al (2017) Type 1 diabetes mellitus. Nat Rev Dis Primers 3(1):17016. https://doi.org/ 10.1038/nrdp.2017.16

2. Buckner JH, Nepom GT (2016) Obstacles and opportunities for targeting the effector $\mathrm{T}$ cell response in type 1 diabetes. $\mathrm{J}$ Autoimmun 71:44-50. https://doi.org/10.1016/j.jaut.2016.02.009

3. Hull CM, Peakman M, Tree TIM (2017) Regulatory T cell dysfunction in type 1 diabetes: what's broken and how can we fix it? Diabetologia 60(10):1839-1850. https://doi.org/10.1007/s00125017-4377-1

4. Creusot RJ, Postigo-Fernandez J, Teteloshvili N (2018) Altered function of antigen-presenting cells in type 1 diabetes: a challenge for antigen-specific immunotherapy? Diabetes 67(8):1481-1494. https://doi.org/10.2337/db17-1564

5. Gagnerault MC, Luan JJ, Lotton C, Lepault F (2002) Pancreatic lymph nodes are required for priming of beta cell reactive $T$ cells in NOD mice. J Exp Med 196(3):369-377. https://doi.org/10.1084/ jem. 20011353

6. Ferraro A, Socci C, Stabilini A et al (2011) Expansion of Th17 cells and functional defects in T regulatory cells are key features of the pancreatic lymph nodes in patients with type 1 diabetes. Diabetes 60(11):2903-2913. https://doi.org/10.2337/db11-0090

7. Kambayashi T, Laufer TM (2014) Atypical MHC class IIexpressing antigen-presenting cells: can anything replace a dendritic cell? Nat Rev Immunol 14(11):719-730. https://doi.org/10.1038/ nri3754

8. Fletcher AL, Malhotra D, Turley SJ (2011) Lymph node stroma broaden the peripheral tolerance paradigm. Trends Immunol 32(1):12-18. https://doi.org/10.1016/j.it.2010.11.002

9. Alexandre YO, Mueller SN (2018) Stromal cell networks coordinate immune response generation and maintenance. Immunol Rev 283(1):77-85. https://doi.org/10.1111/imr.12641

10. Miller CN, Hartigan-O'Connor DJ, Lee MS et al (2013) IL-7 production in murine lymphatic endothelial cells and induction in the setting of peripheral lymphopenia. Int Immunol 25(8):471-483. https://doi.org/10.1093/intimm/dxt012

11. Siegert S, Luther SA (2012) Positive and negative regulation of T cell responses by fibroblastic reticular cells within paracortical regions of lymph nodes. Front Immunol 3:285. https://doi.org/10. 3389/fimmu.2012.00285

12. Hirosue S, Dubrot J (2015) Modes of antigen presentation by lymph node stromal cells and their immunological implications. Front Immunol 6:446. https://doi.org/10.3389/fimmu.2015.00446

13. Tewalt EF, Cohen JN, Rouhani SJ et al (2012) Lymphatic endothelial cells induce tolerance via PD-L1 and lack of costimulation leading to high-level PD-1 expression on CD8 T cells. Blood 120(24):4772-4782. https://doi.org/10.1182/blood-2012-04427013

14. Carpenter DJ, Granot T, Matsuoka N et al (2018) Human immunology studies using organ donors: Impact of clinical variations on immune parameters in tissues and circultion. Am J Transplant 18(1):74-88 
15. Sathaliyawala T, Kubota M, Yudanin N et al (2013) Distribution and compartmentalization of human circulating and tissue-resident memory T cell subsets. Immunity 38(1):187-197

16. Metzger TC, Anderson MS (2011) Control of central and peripheral tolerance by Aire. Immunol Rev 241(1):89-103. https://doi.org/10. 1111/j.1600-065X.2011.01008.x

17. Gardner JM, Metzger TC, McMahon EJ et al (2013) Extrathymic Aire-expressing cells are a distinct bone marrow-derived population that induce functional inactivation of $\mathrm{CD}^{+} \mathrm{T}$ cells. Immunity 39(3):560-572. https://doi.org/10.1016/j.immuni.2013.08.005

18. Postigo-Fernandez J, Farber DL, Creusot RJ (2019) Additional data for 'Phenotypic alterations in pancreatic lymph node stromal cells from human donors with type 1 diabetes and NOD mice'. Mendeley. https://doi.org/10.17632/d9rdzdmvyf.1

19. Kulshrestha D, Yeh LT, Chien MW, Chou FC, Sytwu HK (2017) Peripheral autoimmune regulator induces exhaustion of $\mathrm{CD}^{+}$and $\mathrm{CD}^{+}$effector $\mathrm{T}$ cells to attenuate autoimmune diabetes in nonobese diabetic mice. Front Immunol 8:1128. https://doi.org/10. 3389/fimmu.2017.01128

20. Gardner JM, Devoss JJ, Friedman RS et al (2008) Deletional tolerance mediated by extrathymic Aire-expressing cells. Science 321(5890):843-847. https://doi.org/10.1126/science.1159407

21. Becklund BR, Purton JF, Ramsey C et al (2016) The aged lymphoid tissue environment fails to support naive T cell homeostasis. Sci Rep 6(1):30842. https://doi.org/10.1038/srep30842

22. Cohen JN, Guidi CJ, Tewalt EF et al (2010) Lymph node-resident lymphatic endothelial cells mediate peripheral tolerance via Aireindependent direct antigen presentation. J Exp Med 207(4):681688. https://doi.org/10.1084/jem.20092465

23. Yip L, Creusot RJ, Pager CT, Sarnow P, Fathman CG (2013) Reduced DEAF1 function during type 1 diabetes inhibits translation in lymph node stromal cells by suppressing Eif4g3. J Mol Cell Biol 5(2):99-110. https://doi.org/10.1093/jmcb/mjs052

24. Thayer TC, Pearson JA, De Leenheer E et al (2016) Peripheral proinsulin expression controls low-avidity proinsulin-reactive CD8 T cells in type 1 diabetes. Diabetes 65(11):3429-3439. https://doi.org/10.2337/db15-1649

25. Dubrot J, Duraes FV, Potin L et al (2014) Lymph node stromal cells acquire peptide-MHCII complexes from dendritic cells and induce antigen-specific $\mathrm{CD}^{+} \mathrm{T}$ cell tolerance. J Exp Med 211(6):11531166. https://doi.org/10.1084/jem.20132000

26. Lee J, Epardaud M, Sun J et al (2007) Peripheral antigen display by lymph node stroma promotes T cell tolerance to intestinal self. Nat Immunol 8(2):181-190. https://doi.org/10.1038/ni1427

27. Nichols L, Chen Y, Colella T, Bennett C, Clausen B, Engelhard V (2007) Deletional self-tolerance to a melanocyte/melanoma antigen derived from tyrosinase is mediated by a radio-resistant cell in peripheral and mesenteric lymph nodes. J Immunol 179(2):993-1003. https://doi.org/10.4049/jimmunol.179.2.993
28. Fletcher AL, Lukacs-Kornek V, Reynoso ED et al (2010) Lymph node fibroblastic reticular cells directly present peripheral tissue antigen under steady-state and inflammatory conditions. J Exp Med 207(4):689-697. https://doi.org/10.1084/jem.20092642

29. Yip L, Su L, Sheng D et al (2009) Deafl isoforms control the expression of genes encoding peripheral tissue antigens in the pancreatic lymph nodes during type 1 diabetes. Nat Immunol 10(9): 1026-1033. https://doi.org/10.1038/ni.1773

30. Malhotra D, Fletcher AL, Astarita J et al (2012) Transcriptional profiling of stroma from inflamed and resting lymph nodes defines immunological hallmarks. Nat Immunol 13(5):499-510. https:// doi.org/10.1038/ni.2262

31. Hahnlein JS, Nadafi R, de Jong T et al (2018) Impaired lymph node stromal cell function during the earliest phases of rheumatoid arthritis. Arthritis Res Ther 20(1):35. https://doi.org/10.1186/s13075018-1529-8

32. Valencia J, Jimenez E, Martinez VG et al (2017) Characterization of human fibroblastic reticular cells as potential immunotherapeutic tools. Cytotherapy 19(5):640-653. https://doi.org/10.1016/j.jcyt. 2017.01.010

33. Lucas ED, Finlon JM, Burchill MA et al (2018) Type 1 IFN and PD-L1 coordinate lymphatic endothelial cell expansion and contraction during an inflammatory immune response. J Immunol 201(6):1735-1747. https://doi.org/10.4049/jimmunol.1800271

34. Osum KC, Burrack AL, Martinov T et al (2018) Interferon-gamma drives programmed death-ligand 1 expression on islet $\beta$ cells to limit $\mathrm{T}$ cell function during autoimmune diabetes. Sci Rep 8(1): 8295. https://doi.org/10.1038/s41598-018-26471-9

35. Baptista AP, Roozendaal R, Reijmers RM et al (2014) Lymph node stromal cells constrain immunity via MHC class II self-antigen presentation. eLife 3. https://doi.org/10.7554/eLife.04433

36. Dubrot J, Duraes FV, Harle G et al (2018) Absence of MHC-II expression by lymph node stromal cells results in autoimmunity. Life Sci Alliance 1(6):e201800164. https://doi.org/10.26508/lsa. 201800164

37. Kumar V, Dasoveanu DC, Chyou S et al (2015) A dendritic-cellstromal axis maintains immune responses in lymph nodes. Immunity 42(4):719-730. https://doi.org/10.1016/j.immuni.2015. 03.015

38. Cire S, Da Rocha S, Ferrand M, Collins MK, Galy A (2016) In vivo gene delivery to lymph node stromal cells leads to transgenespecific CD8+ T cell anergy in mice. Mol Ther 24(11):19651973. https://doi.org/10.1038/mt.2016.168

Publisher's note Springer Nature remains neutral with regard to jurisdictional claims in published maps and institutional affiliations. 